\title{
Toward Green Communications Using Semi-Blind Channel Estimation
}

\author{
Abdelhamid Ladaycia*, Anissa Mokraoui*, Karim Abed-Meraim ${ }^{\dagger}$ and Adel Belouchrani ${ }^{\ddagger}$ \\ * L2TI, Institut Galilée, Université Paris 13 Sorbonne Paris Cité, France \\ \{abdelhamid.ladaycia, anissa.mokraoui\}@univ-paris13.fr \\ ${ }^{\dagger}$ PRISME, Université d'Orléans, France \\ karim.abed-meraim@univ-orleans.fr \\ ${ }^{\ddagger}$ Electrical Engineering Department/LDCCP, Ecole Nationale Polytechnique, Algeria \\ adel.belouchrani@enp.edu.dz
}

\begin{abstract}
In most Multiple-Input Multiple-Output Orthogonal Frequency Division Multiplexing (MIMO-OFDM) systems, channel estimation is required for equalization and symbol detection. It often exploits the specified pilot symbols consuming not only a large part of the throughput but also significant power resources. This paper quantifies the theoretical maximum power reduction of the transmitted pilots when semiblind channel estimator is deployed while ensuring the same pilot-based channel estimation performance for BPSK/QPSK data models and a block-type pilot arrangement as specified in the IEEE 802.11n standard. A Least Square Decision Feedback (LS-DF) semi-blind channel estimator is then considered showing that a reduction of $76 \%$ of the pilot's power is obtained compared to the LS pilot-based estimator for the same channel estimation performance.
\end{abstract}

\section{Introduction}

Channel estimation is of paramount importance to equalization and symbol detection problems in most wireless communications systems. Many approaches have been developed and can be classified into two main categories.

The first one concerns blind channel estimation methods which have been extensively studied and are based on the statistical properties of the transmitted symbols (e.g. [1]).

The second one, adopted in most communications standards [2], relies on the insertion of pilots in the physical packet according to a given arrangement type (block, comb or lattice) [3], [4]. However these pilots consume not only a large part of throughput but also significant power resources. This becomes even more important for future communications systems such as massive-MIMO systems. Indeed the explosive growth of high data rate applications where the corresponding energy consumption is also growing at a staggering rate has urged for an intensive research work on green communications to protect our environment and cope with global warming [5]. In [6], the throughput problem has been investigated for Single-Input Multiple-Output Orthogonal Frequency Division Multiplexing (SIMO-OFDM) systems. In [7], authors present the state-of-the-art of the green communications methods. Antenna selection using beamforming algorithm is proposed in [8].
This paper suggests an unusual approach to reduce the consumed power making the most of the advantages of semi-blind channel estimation approaches. The underlying idea consists of removing pilot samples which are replaced by zero-samples while ensuring the same performance as pilot-based channel estimation approaches. The maximal reduction of the theoretical transmitted pilot's power is first addressed when semi-blind approaches are deployed instead of pilot-based approaches for the same estimation performance. To do so, the theoretical limit channel estimation performance, based on the analytical Cramér Rao Bound (CRB), is considered. The real gain in terms of pilot's power reduction at the transmitter is then evaluated when Least Square Decision Feedback (LS-DF) semi-blind channel estimator is used. In addition, the overconsumption at the receiver is evaluated and discussed.

This paper is organized as follows. Section 2 presents the considered MIMO-OFDM wireless system model. Section 3 introduces the analytical expressions of the CRB for semiblind channel estimation for BPSK/QPSK data models and when block-type pilot arrangement is considered. The LSDF semi-blind channel estimator and its computational cost are developed in section 4.1. Simulation results are discussed in section 5. Finally, section 6 concludes the paper.

\section{MIMO-OFDM wireless system}

This section presents the MIMO-OFDM wireless system. It is composed of $N_{t}$ transmit antennas and $N_{r}$ receive antennas. The transmitted signal is assumed to be an OFDM one, composed of $K$ samples (sub-carriers) and $L$ Cyclic Prefix (CP) samples. The CP length is assumed to be greater or equal to the maximum multipath channel delay denoted $N$ (i.e. $N \leq L$ ). After removing the $\mathrm{CP}$ and taking the $K$-point FFT, the received signal, denoted $\mathbf{y}$, is given by:

$$
\mathbf{y}=\sum_{i=1}^{N_{t}} \boldsymbol{\Lambda}_{i} \mathbf{x}_{i}+\mathbf{v},
$$

where $\mathbf{x}_{i}$ is the transmitted OFDM symbol by the $i$-th transmitter, and $\mathbf{y}=\left[\mathbf{y}_{1}^{T} \cdots \mathbf{y}_{N_{r}}^{T}\right]^{T}$ is the received signal by the $N_{r}$ receiver. The noise $\mathbf{v}=\left[\mathbf{v}_{1}^{T} \cdots \mathbf{v}_{N_{r}}^{T}\right]^{T}$ is assumed to 
be additive independent white Circular Complex Gaussian (CCG) satisfying $E\left[\mathbf{v}(k) \mathbf{v}(i)^{H}\right]=\sigma_{\mathbf{v}}^{2} \mathbf{I}_{K} \delta_{k i} ;(.)^{H}$ being the Hermitian operator; $\sigma_{\mathbf{v}}^{2}$ the noise variance; $\mathbf{I}_{K}$ the identity matrix of size $K \times K$ and $\delta_{k i}$ the Dirac operator. The matrices $\boldsymbol{\Lambda}_{i}$ are given by:

$$
\boldsymbol{\Lambda}_{i}=\left[\boldsymbol{\Lambda}_{i, 1} \cdots \boldsymbol{\Lambda}_{i, N_{r}}\right]^{T} \text { where } \boldsymbol{\Lambda}_{i, r}=\operatorname{diag}\left\{\mathbf{W} \mathbf{h}_{i, r}\right\},
$$

where $\mathbf{W}$ is a matrix containing the $N$ first columns of $\mathbf{F}$, which represents the $K$-point Fourier transform matrix. The $N \times 1$ vector, representing propagation channel taps between the $i$-th transmit antenna and the $r$-th receive antenna, is denoted by $\mathbf{h}_{i, r}$.

Equation (1) can be rewritten in a matrix form:

$$
\mathbf{y}=\Lambda \mathbf{x}+\mathbf{v},
$$

where $\boldsymbol{\Lambda}=\left[\boldsymbol{\Lambda}_{1} \cdots \boldsymbol{\Lambda}_{N_{t}}\right]$, and $\mathbf{x}=\left[\mathbf{x}_{1}^{T} \cdots \mathbf{x}_{N_{t}}^{T}\right]^{T}$.

For convenience equation (3) is rewritten as:

$$
\mathbf{y}=\tilde{\mathbf{X}} \mathbf{h}+\mathbf{v}
$$

The global channel propagation vector of size $N_{r} N_{t} N \times$ 1 is $\mathbf{h}=\left[\mathbf{h}_{1}^{T} \cdots \mathbf{h}_{N_{r}}^{T}\right]^{T}$, where $\mathbf{h}_{r}=\left[\mathbf{h}_{1, r}^{T} \cdots \mathbf{h}_{N_{t}, r}^{T}\right]^{T}$. $\mathbf{X}_{D_{i}}=\operatorname{diag}\left\{\mathbf{x}_{i}\right\}$ is a diagonal matrix of size $K \times K$; $\mathbf{X}=\left[\mathbf{X}_{D_{1}} \mathbf{W} \cdots \mathbf{X}_{D_{N_{t}}} \mathbf{W}\right]$ of size $K \times N N_{t}$.

Where $\tilde{\mathbf{X}}=\mathbf{I}_{N_{r}} \otimes \mathbf{X}$ is a matrix of size $N_{r} K \times N N_{t} N_{r}$ and $\otimes$ refers to the Kronecker product.

In the sequel, to take into account the time index (ignored in equations (1), (3) and (4)), we will refer to the $t$-th OFDM symbol by $\mathbf{y}(t)$ instead of $\mathbf{y}$.

\section{CRB for semi-blind channel estimation}

This section introduces the CRB expressions for semiblind channel estimation. Thanks to CRB, the theoretical limit power reduction of the transmitted pilots will be deduced and discussed in section 5. A block-type pilot arrangement and two data signal models (i.i.d. BPSK/QPSK signals) are considered. Data and noise are assumed to be independent. Moreover the mobile stations are assumed to be perfectly synchronized.

First consider pilot-based channel estimation approach. Denote $\mathbf{J}_{\Theta \Theta}^{p}$ the Fisher Information Matrix (FIM) where $\Theta$ is the unknown parameter vector containing the channel vector i.e. $\Theta=\mathbf{h}^{T}$ to be estimated. The FIM $\mathbf{J}_{\Theta \Theta}^{p}$ is given by (see [6]):

$$
\mathbf{J}_{\Theta \Theta}^{p}=\frac{\tilde{\mathbf{X}}_{p}^{H} \tilde{\mathbf{X}}_{p}}{\sigma_{\mathbf{v}}^{2}} .
$$

The CRB for the mean squares estimation error (MSE) of vector $\mathbf{h}$, denoted $C R B_{O P}$, is then deduced as follows:

$$
C R B_{O P}=\sigma_{\mathbf{v}}^{2} \operatorname{tr}\left\{\left(\tilde{\mathbf{X}}_{p}^{H} \tilde{\mathbf{X}}_{p}\right)^{-1}\right\}
$$

where $\tilde{\mathbf{X}}_{p}=\left[\tilde{\mathbf{X}}(1)^{T} \cdots \tilde{\mathbf{X}}\left(N_{p}\right)^{T}\right]^{T}$ with $N_{p}$ pilot OFDM symbols.
For semi-blind channel estimation approach, the FIM is divided into pilot part (i.e. $\mathbf{J}_{\Theta \Theta}^{p}$ ) and data part (i.e. $\mathbf{J}_{\Theta \Theta}^{d}$ ):

$$
\mathbf{J}_{\Theta \Theta}=\mathbf{J}_{\Theta \Theta}^{p}+\mathbf{J}_{\Theta \Theta}^{d} .
$$

For BPSK/QPSK data models, the CRB computation in a MIMO-OFDM system, is prohibitive [9]. In [10], we proposed a realistic approximation to bypass the high complexity of the exact BPSK/QPSK FIM computation. In $\left(N_{t} \times N_{r}\right)$ MIMO-OFDM system, the likelihood function is given as a mixture of $Q^{N_{t}}$ Gaussian pdfs:

$$
p\left(\mathbf{y}_{(k)}, \Theta\right)=\frac{1}{Q^{N_{t}}} \sum_{q=1}^{Q^{N_{t}}} \frac{1}{\left(\pi \sigma_{\mathbf{v}}^{2}\right)^{N_{r}}} e^{-\left\|\mathbf{y}_{(k)}-\boldsymbol{\Lambda}_{(k)} \mathbf{x}_{q}\right\|^{2} / \sigma_{\mathbf{v}}^{2}},
$$

where $Q=2$ and the entries of $x_{k}$ belong to \pm 1 (respectively $Q=4$ and the entries of $x_{k}$ belong to $\pm \sqrt{2} / 2 \pm i \sqrt{2} / 2$ ) for BPSK (respectively QPSK) modulation. $\mathbf{y}_{(k)}=\left[y_{1, k} \cdots y_{N_{r}, k}\right]^{T}$ represents the received signal at the $k$-th sub-carrier and $\boldsymbol{\Lambda}_{(k)}$ is the $k$-th component of the FFT of $\mathbf{h}$ given as: $\boldsymbol{\Lambda}_{(k)}=\left[\boldsymbol{\Lambda}_{(k), 1}, \cdots, \boldsymbol{\Lambda}_{(k), N_{t}}\right]$ where $\boldsymbol{\Lambda}_{(k), i}=\operatorname{diag}\left\{\left(\mathbf{W h}_{i, 1}\right)_{k}, \cdots,\left(\mathbf{W h}_{i, N_{r}}\right)_{k}\right\}$.

The total FIM is expressed as follows:

$$
\mathbf{J}_{\Theta \Theta}^{d}=N_{d} \sum_{k=1}^{K} \mathbf{J}_{\Theta \Theta}^{d}(k),
$$

where $N_{d}$ is the number of data OFDM symbols. The FIM at the $k$-th sub-carrier $\mathbf{J}_{\Theta \Theta}^{d}(k)$ is provided in [10]:

$$
\mathbf{J}_{\Theta \Theta}^{d}(k)=\frac{1}{\sigma_{\mathbf{v}}^{2} Q^{N_{t}}} \sum_{q=1}^{Q^{N_{t}}}\left(\frac{\partial \boldsymbol{\Lambda}_{(k)} \mathbf{x}_{q}}{\partial \boldsymbol{\Theta}^{*}}\right)^{H}\left(\frac{\partial \boldsymbol{\Lambda}_{(k)} \mathbf{x}_{q}}{\partial \boldsymbol{\Theta}^{*}}\right) .
$$

\section{LS-DF semi-blind channel estimation algo- rithm}

In the sequel, we will use the derived semi-blind CRB to evaluate the maximum shortening of the pilot sequence that can be afforded without affecting the channel estimaion quality. This will be used to compute the power saving due to this shortening at the transmitter side. For comparison fairness, we need to evaluate to power consumption increase at the receiver side due to the use of a more elaborate semiblind estimation algorithm. For this reason, we introduce in this section a semi-blind estimation method that has the advantages of simplicity and effectiveness (i.e. it reaches the CRB for moderate and high SNRs).

\subsection{Main steps of the LS-DF algorithm}

The LS-DF channel estimation algorithm is considered as a LS estimator which incorporates the feedback equalizer. Traditionally the LS-DF algorithm re-injects the estimated signal as a feedback to the equalizer stage to enhance the estimation performance of the transmitted data. This process is iterated several times.

Instead of using the LS-DF algorithm in its original version, this paper exploits this algorithm as a semi-blind 
channel estimator since the estimated data at the previous stage are now considered as "pilots" when the algorithm re-estimates the channel taps according to the LS channel estimation as illustrated in Figure 1.

According to the system model represented by equation (4), the conventional LS pilot-based channel estimation is expressed by (for more details see [4]):

$$
\hat{\mathbf{h}}_{o p}=\left(\tilde{\mathbf{X}}_{p}^{H} \tilde{\mathbf{X}}_{p}\right)^{-1} \tilde{\mathbf{X}}_{p}^{H} \mathbf{y} .
$$

The LS channel estimation performance is widely discussed in literature. It has been shown that the mean squares error (MSE) of this estimator reaches the $C R B_{O P}$. Therefore the $M S E_{O P}$ is given by:

$$
M S E_{O P}=\sigma_{\mathbf{v}}^{2} \operatorname{tr}\left\{\left(\tilde{\mathbf{X}}_{p}^{H} \tilde{\mathbf{X}}_{p}\right)^{-1}\right\} .
$$

Moreover when the training sequences $\mathbf{x}_{p}$ are orthogonal, $\left(\tilde{\mathbf{X}}_{p}^{H} \tilde{\mathbf{X}}_{p}\right)$ is equal to $\sigma_{p}^{2} \mathbf{I}_{N N_{t} N_{r}}$ and the MSE is minimal. After estimating the channel (i.e. $\hat{\mathbf{h}}_{o p}$ ), the Zero-Forcing

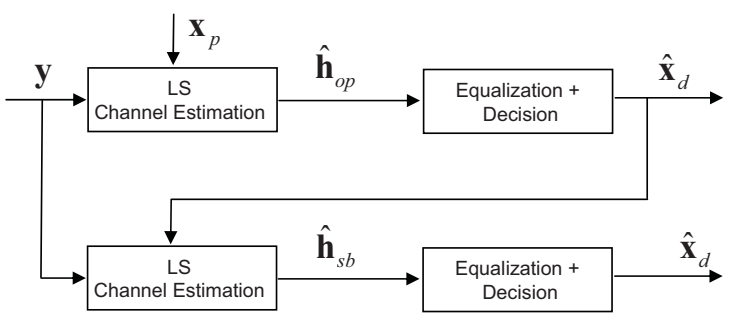

Figure 1. LS-DFE semi-blind channel estimation approach.

(ZF) equalizer is adopted to estimate the transmitted signal. It refers to a form of linear equalization algorithm often used in communications systems. It applies the inverse of the channel frequency response $\hat{\Lambda}^{\#}$ to the received signal where $\#$ denotes the pseudo inverse matrix, and $\hat{\Lambda}$ is the channel frequency response of $\hat{\mathbf{h}}_{o p}$ calculated as in section 2 . The equalized signal, denoted $\mathbf{x}_{z f}$, is then deduced:

$$
\mathbf{x}_{z f}=\hat{\boldsymbol{\Lambda}}^{\#} \mathbf{y}=\hat{\boldsymbol{\Lambda}}^{\#} \boldsymbol{\Lambda} \mathbf{x}+\mathbf{v} .
$$

After that, a hard decision is taken on the equalized signal to estimate the transmitted signal $\hat{\mathbf{x}}$. The new training sequences become:

$$
\mathbf{x}_{p}=\left[\mathbf{x}_{p}^{T} \hat{\mathbf{x}}^{T}\right]^{T} .
$$

Based on equation (11), the channel taps are then estimated ( $\hat{\mathbf{h}}_{\mathrm{sb}}$ in Figure 1). The ZF equalizer, given by equation (13), estimates the signal $\mathbf{x}_{z f}$ on which a hard decision is taken to estimate the transmitted data $\hat{\mathbf{x}}_{\mathbf{d}}$.

\subsection{Computational cost comparison of LS and LS- DF algorithms}

This section compares the computational cost of the LS-DF semi-blind channel estimator to the LS pilot-based channel estimation. The computational cost is evaluated in terms of real number of flops (i.e. number of multiplications plus number of additions).

At the receiver, the number of flops consumed by LS pilot-based channel estimation algorithm is deduced from equation (11) where $\tilde{\mathbf{X}}_{p}$ and $\mathbf{y}$ are of size $N_{p} N_{r} K \times N_{t} N_{r} N$ and $N_{p} N_{r} K \times 1$ respectively. The details of the number of flops required to estimate $\hat{\mathbf{h}}_{o p}$ are listed in Table 1 .

At the receiver, the flops consumed by the LS-DF algorithm is equal to the flops due to the equalizer/decision stage added to the flops required to estimate $\hat{\mathbf{h}}_{s b}$ and $\hat{\mathbf{h}}_{o p}$ (see Table 1). Note that the flops required for the equalizer/decision stage can be easily compensated by the reduction of the flops due to the removed pilots from the initial training sequence, this will be discussed in simulation results (90\% samples of the initial training sequence are removed). Therefore the LS-DF semi-blind channel estimator consumes $\Delta_{\text {Flops }}$ more flops than the LS pilot-based channel estimator:

$$
\begin{aligned}
& \Delta_{\text {flops }}=2\left(N_{t} N_{r} N\right)^{3}+4\left(N_{t} N_{r} N\right)^{2}\left(N_{d} N_{r} K\right) \\
& +\left(N_{t} N_{r} N\right)\left(N_{d} N_{r} K\right)-\left(N_{t} N_{r} N\right)^{2}-\left(N_{t} N_{r} N\right) .
\end{aligned}
$$

In [11], the authors investigate the relationship between the flops number and the corresponding consumed power denoted Flops per Watt (Flops/Watt). It is then possible to measure the equivalent consumed power in Watts. Depending on the functional characteristics of the processor, the consumed power per Watt is between 5 and 100 GFlops/Watt. If $P$ is the consumed power, given in GFlops/Watt, the consumed power associated to $\Delta_{\text {Flops }}$ can be deduced as follows:

$$
\Delta_{\text {Power }}=\frac{\Delta_{\text {flops }}}{P} 10^{-6} \mathrm{mWatt}
$$

\begin{tabular}{|c|c|}
\hline Operation & Number of flops \\
\hline$\tilde{\mathbf{X}}_{p}^{H} \tilde{\mathbf{X}}_{p}$ & $2\left(N_{t} N_{r} N\right)^{2} N_{p} N_{r} K-\left(N_{t} N_{r} N\right)^{2}$ \\
\hline$\left(\tilde{\mathbf{X}}_{p}^{H} \tilde{\mathbf{X}}_{p}\right)^{-1}$ & $2\left(N_{t} N_{r} N\right)^{3}$ \\
\hline$\left(\tilde{\mathbf{X}}_{p}^{H} \tilde{\mathbf{X}}_{p}\right)^{-1} \tilde{\mathbf{X}}_{p}^{H}$ & $2\left(N_{t} N_{r} N\right)^{2} N_{p} N_{r} K-\left(N_{t} N_{r} N\right) N_{p} N_{r} K$ \\
\hline$\left(\tilde{\mathbf{X}}_{p}^{H} \tilde{\mathbf{X}}_{p}\right)^{-1} \tilde{\mathbf{X}}_{p}^{H} \mathbf{y}$ & $2\left(N_{t} N_{r} N\right) N_{p} N_{r} K-\left(N_{t} N_{r} N\right)$ \\
\hline & $2\left(N_{t} N_{r} N\right)^{3}+4\left(N_{t} N_{r} N\right)^{2} N_{p} N_{r} K$ \\
& $+\left(N_{t} N_{r} N\right) N_{p} N_{r} K-\left(N_{t} N_{r} N\right)^{2}$ \\
$\hat{\mathbf{h}}_{o p}$ & $-\left(N_{t} N_{r} N\right)$ \\
& $4\left(N_{t} N_{r} N\right)^{2}\left(\left(N_{p}+N_{d}\right) N_{r} K\right)+$ \\
& $\left(N_{t} N_{r} N\right)\left(\left(N_{p}+N_{d}\right) N_{r} K\right)+$ \\
& $2\left(N_{t} N_{r} N\right)^{3}-\left(N_{t} N_{r} N\right)^{2}-\left(N_{t} N_{r} N\right)$ \\
\hline & $2 N_{t} N_{r} N_{d} K^{2}+2\left(N_{r} K\right)^{2} N_{t} K+$ \\
& $2\left(N_{t} K\right)^{3}-\left(N_{r} K\right)^{2}$ \\
& $-N_{t} N_{r} K^{2}-N_{t} N_{d} K$ \\
\hline \multirow{3}{*}{ Flops $s_{E q}$} & TABLE 1. FLOPS NUMBER. \\
\end{tabular}

\section{Performance analysis and discussions}

This section analyzes and quantifies the transmitted power that can be reduced when semi-blind channel estimation approach is deployed while maintaining the same performance as LS pilot-based channel estimation approach.

The considered MIMO-OFDM wireless system is related to the IEEE 802.11n standard [2]. The training sequences correspond to those specified by the standard. In the legay 
preamble (i.e. 802.11a) two identical fields named LTF (Long Training Field) are dedicated to channel estimation. Each field (or pilot) is represented by one OFDM symbol ( $K=64$ samples) where a $\mathrm{CP}$ ( $L=16$ samples) is added at its front. In the High Throughput preamble, a set of identical fields named High Throughput Long Training fields (HT-LTF) are specified and represented by one OFDM symbol ( $K=64$ samples) with a CP (16 samples). These fields (or pilots) are specified to MIMO channel estimation. Their number depends on the number of transmit antennas $\left(N_{t}\right)$. Since in this paper $N_{t}=3$, four $\left(N_{p}^{H T-L T F}=4\right)$ HT-LTF pilot OFDM symbols are used (see [2] for details). Therefore the training sequence length is equal to $N_{p}=N_{p}^{L T F}+N_{p}^{H T-L T F}$. The data field is represented by a set of OFDM symbols depending on the length of the transmitted packet $\left(N_{d}\right)$. Simulation parameters are summarized in Table 2.

The Signal to Noise Ratio associated with pilots at the reception is defined as $S N R_{p}=\frac{\left\|\boldsymbol{\Lambda} \mathbf{x}_{p}\right\|^{2}}{N_{r} N_{p} K \sigma_{\mathbf{v}}^{2}}$. The signal to noise ratio $S N R_{d}$ associated with data is given (in $\mathrm{dB}$ ) by: $S N R_{d}=S N R_{p}-\left(P x_{p}-P x_{d}\right)$ where $P x_{p}$ (respectively $P x_{d}$ ) is the power of pilots (respectively data) (both in $\mathrm{dB}$ ).

\subsection{Theoretical limit pilot's power reduction}

This section analyzes the maximum pilot's power reduction evaluated from the theoretical limit bound performance of the semi-blind channel estimation approach.

The transmitted pilot's power is reduced in such a way that semi-blind approach achieves the same performance as pilot-based channel estimation approach (i.e. $C R B_{O P}$ ). To do so, the proposed strategy replaces the removed pilot samples by zero-samples leading therefore to a reduction of the average pilot's transmitted power(or equivalently to the transmitted energy).

Figure 2 provides the CRB for semi-blind channel estimation versus the reduced pilot's power for a given $S N R_{p}=12 \mathrm{~dB}$. The horizontal line represents the CRB for pilot-based channel estimation and is considered as the reference to be reached. Only $8 \%$ of pilot's power is retained (i.e. $185 \mathrm{~mW}$ are reduced). These results show clearly that semi-blind estimation in MIMO-OFDM system brings a significant gain in terms of the transmitted pilot's energy reduction.

Figure 3 shows the impact of the number of data OFDM symbols on the pilot's transmitted power (in percentage) for a given $S N R_{p}=12 \mathrm{~dB}$. When the number of data OFDM symbols increases, the percentage of the reduced pilot's power becomes more significant. Note that the results observed in Figure 2 can be deduced from Figure 3 when the number of data OFDM symbols is equal to 40 .

\subsection{LS-DF performance in terms of power con- sumption}

This section investigates the energy balance of the complete system (transmitter and receiver), namely the power deployed by the transmitter and that consumed by the receiver when the LS-DF algorithm is adopted.

The curves in Figure 4 present the Normalized Root Mean square Error (NRMSE) of LS and LS-DF estimators versus the $S N R_{p}$. Note that for the $\mathbf{h}_{S B}^{B P S}, \mathbf{h}_{S B}^{Q P S K} \mathrm{LS}-$ DF reaches the $C R B_{S B}^{B P S K}, C R B_{S B}^{Q P S K}$ at height SNR, and gives better results compared to the LS pilot-based approach $\left(\mathbf{h}_{O P}\right)$ from $S N R_{p}=2 \mathrm{~dB}$.

\begin{tabular}{|c|c|}
\hline Parameters & Specifications \\
\hline Channel model & Cost 207 \\
\hline Number of transmit antennas & $N_{t}=3$ \\
\hline Number of receive antennas & $N_{r}=4$ \\
\hline Channel length & $N=4$ \\
\hline Number of LTF pilot OFDM symbols & $N_{p}^{L T F}=2$ \\
\hline Number of HT-LTF pilot OFDM symbols & $N_{p}^{H T-L T F}=4$ \\
\hline Number of data OFDM symbols & $N_{d}=40$ \\
\hline Pilot signal power & $P_{x_{p}}=23 \mathrm{dBm}$ \\
\hline Data signal power & $P_{x_{d}}=20 \mathrm{dBm}$ \\
\hline Number of sub-carriers & $K=64$ \\
\hline Consumed power (GFlops/Watt) & $P=5$ \\
\hline
\end{tabular}

TABLE 2. SiMULATION PARAMETERS.

Figure 5 presents the transmitted pilot's power versus the $S N R_{p}$. The higher the $S N R_{p}$ is, the lower transmitted pilot's power is in favor of the LS-DF semi-blind estimator.

Figure 6 provides the NRMSE of the LS-DF estimator versus the reduced pilot's power (in percentage) for a given $S N R_{p}=12 \mathrm{~dB}$ (with $N_{d}=40$ ). The pilot's power is reduced in such a way that the LS-DF estimator performance $\left(\mathbf{h}_{S B}^{B P S K}, \mathbf{h}_{S B}^{Q P S K}\right)$ reaches the same performance as the LS pilot-based estimator. For BPSK data model, only $49 \mathrm{~mW}$ is required instead of $200 \mathrm{~mW}(100 \%)$ when pilot-based channel estimation is used (i.e. a reduction of $76 \%$ ). For QPSK data model, 74\% of the pilot's power is also reduced. Although the LS-DF algorithm leads to an overconsumption of the energy at the receiver side since more operations are required (see equation (15), $\Delta_{\text {flops }}=94863360$ Flops, equivalent to $\Delta_{\text {Power }}=19 \mathrm{~mW}$ ), the complete system (i.e. transmitter and receiver) saves $66 \%$ (i.e. $132 \mathrm{~mW}$ ). The flops due to the equalization stage, assumed to compensate the flops associated to the removed pilots (assumption in section 4.2), are equivalent to Flops eq $=3233792$ Flops. While 2924976 Flops are due to the removed pilots. The flops difference is 308816 Flops and is in fact negligible (308816 $<<\Delta_{\text {flops }}$ equivalent to $0.061 \mathrm{~mW}$ ) confirming the assumption. Therefore the global MIMO-OFDM system (i.e. transmitter and receiver) saves $65,97 \%$ i.e. $131.94 \mathrm{~mW}$ of power consumption.

\section{Conclusion}

This paper focused on the power reduction problem in a MIMO-OFDM wireless system specifically during the channel estimation stage. The paper proposed to deploy semiblind channel estimation approach allowing the transmitter to reduce the number of samples in the training sequence while ensuring the same estimation performance as pilotbased channel estimation approach. The maximum theoretical reduction of the pilot's power consumption, based on 
the CRB for semi-blind channel estimation approach, is first investigated for the IEEE 802.11n MIMO-OFDM system with BPSK and QPSK data models. Simulation results, for the same channel estimation performance, show clearly a significant reduction of the pilot's power equivalent to $76 \%$ when LS-DF semi-blind channel estimation is deployed instead of the LS pilot-based channel estimation. A global power reduction of $65,97 \%$ is possible for the complete wireless MIMO-OFDM system.

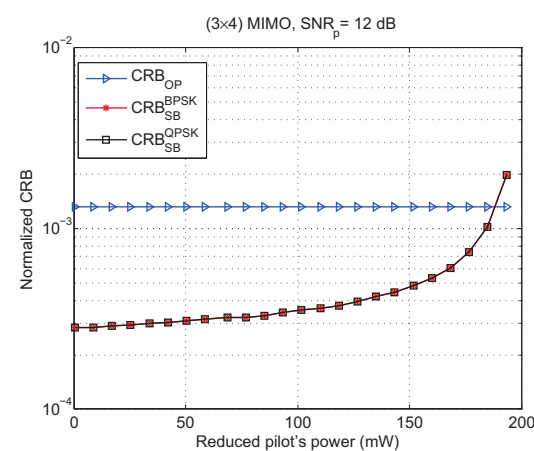

Figure 2. Normalized CRB versus the reduced power $\left(S N R_{p}=12 \mathrm{~dB}\right)$.

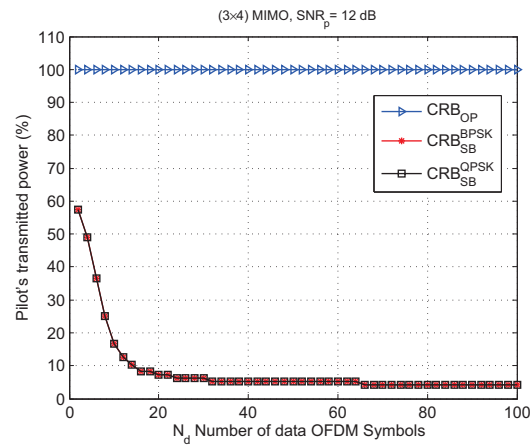

Figure 3. Percentage of the transmitted pilot's power versus the number of data OFDM symbols $N_{d}\left(S N R_{p}=12 \mathrm{~dB}\right)$.

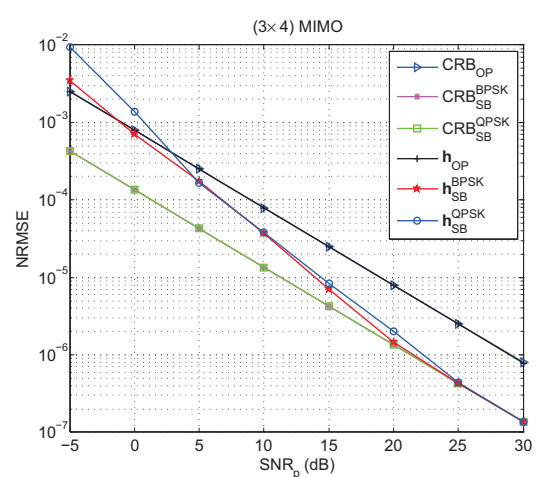

Figure 4. NRMSE of LS and LS-DF estimators versus $S N R_{p}$.

\section{References}

[1] L. Tong, G. Xu, and T. Kailath, "Blind identification and equalization based on second-order statistics: a time domain approach," Information Theory, IEEE Trans. on, vol. 40, no. 2, pp. 340-349, Mar 1994.

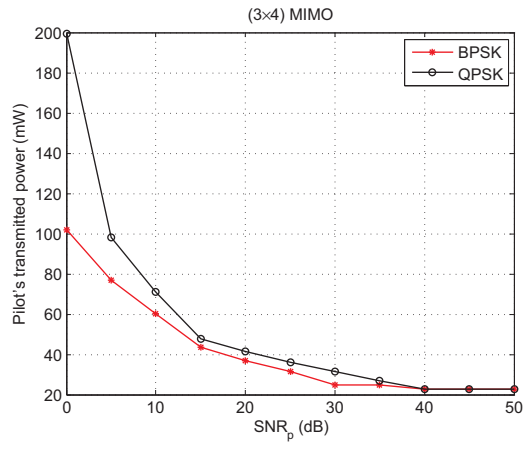

Figure 5. Transmitted pilot's power versus $S N R_{p}$.

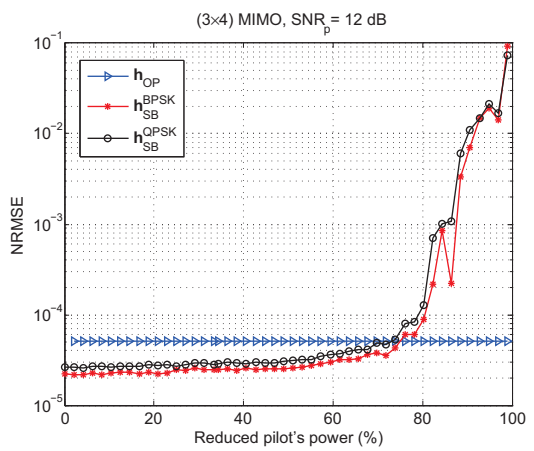

Figure 6. NRMSE of the LS-DF channel estimator versus the percentage of the reduced pilot's power $\left(S N R_{p}=12 \mathrm{~dB}\right)$.

[2] R. S. E. Perahi, Next Generation Wireless LANS. New York, USA: Cambridge University Press, 2008.

[3] W. Zhang, X. G. Xia, and P. C. Ching, "Optimal training and pilot pattern design for OFDM systems in Rayleigh fading," IEEE Trans. on Broadcasting, vol. 52, no. 4, pp. 505-514, Dec 2006.

[4] G. B. Jency and S. J. Gladwin, "Channel estimation using optimized pilot placement in OFDM system," in Commun. and Signal Process. (ICCSP), April 2014.

[5] Y. Chen, S. Zhang, S. Xu, and G. Y. Li, "Fundamental trade-offs on green wireless networks," IEEE Commun. Magazine, June 2011.

[6] A. Ladaycia, A. Mokraoui, K. Abed-Meraim, and A. Belouchrani, "What semi-blind channel estimation brings in terms of throughput gain ?" in 2016 10th International Conference on Signal Processing and Communication Systems (ICSPCS), Dec 2016, pp. 1-6.

[7] D. Feng, C. Jiang, G. Lim, L. J. Cimini, G. Feng, and G. Y. Li, "A survey of energy-efficient wireless communications," IEEE Commun. Surveys Tutorials, vol. 15, no. 1, pp. 167-178, 2013.

[8] K. Qian and W. Q. Wang, "Energy-efficient antenna selection in green mimo relaying communication systems," Journal of Communications and Networks, vol. 18, no. 3, pp. 320-326, June 2016.

[9] J. P. Delmas and H. Abeida, "Stochastic cramer-rao bounds of doa estimates for BPSK and QPSK modulated signals," in ICASSP 2004.

[10] A. Ladaycia, A. Mokraoui, K. Abed-Meraim, and A. Belouchrani, "Performance analysis of semi-blind channel estimation in massive MIMO-OFDM communications systems," in IEEE trans. on wireless communications (Accepted with minor corrections), May 2017.

[11] T. Dong, V. Dobrev, T. Kolev, R. Rieben, S. Tomov, and J. Dongarra, "A step towards energy efficient computing: Redesigning a hydrodynamic application on cpu-gpu," in 2014 IEEE 28th Int. Parallel and Distributed Processing Symposium, May 2014, pp. 972-981. 